\title{
Einfluß verschiedenartig substituierter Cyclopentadienylliganden auf die Chemie schwefelreicher Organometallkomplexe mit Metallen der 6. Nebengruppe
}

\author{
Henri Brunner, Roland Graßl, Walter Meier, Joachim Wachter \\ Institut für Anorganische Chemie der Universität Regensburg, Universitätsstraße 31, W-8400 Regensburg \\ (Deutschland)
}

\author{
Bernd Nuber und Manfred L. Ziegler \\ Anorganisch-chemisches Institut der Universität Heidelberg, Im Neuenheimer Feld 270, W-6900 Heidelberg \\ (Deutschland)
}

(Eingegangen den 14. Januar 1992)

\begin{abstract}
The reaction of the differently substituted cyclopentadienyl complexes $L_{2} M_{2}(C O)_{4}$ with sulfur is investigated with respect to the ligand $L$ (etmcp $=$ ethyltetramethylcyclopentadienyl, $t b c p=t$ butylcyclopentadienyl, ipcp = isopropylcyclopentadienyl) and the metal $\mathrm{M}(\mathrm{Cr}, \mathrm{Mo}, \mathrm{W})$. For $\mathrm{M}=\mathrm{Cr}$ the only isolated product is etmcp $\mathrm{Cr}_{2} \mathrm{~S}_{5}$. For $\mathrm{M}=$ Mo three isomers $A, D, E$ of composition $\mathbf{L}_{2} \mathrm{Mo}_{2} \mathrm{~S}_{4}$ ( $L=$ ipcp, etmcp) are formed whereas only two isomers (A, D) exist for $\mathrm{L}=$ tbcp. Only two isomers (D, E) of composition $L_{2} W_{2} S_{4}$ are formed in the system $L_{2} W_{2}(C O)_{4} / S_{8}$, a further product is $L_{2} W_{2}(C O)_{2} S_{3}$ for $L=$ etmcp. Structural characterisation of all compounds was achieved by $I R,{ }^{1} H$, and ${ }^{95} \mathrm{Mo} N M R$ spectroscopy, whereas the structures of $s y n$-etmcp ${ }_{2} M_{2}(\mu-S){ }_{2} S_{2}(M=M o, W)$ were determined by $\mathrm{X}$-ray crystallography, the result of which allows the correction of a previous structure assignment. It is further shown that a thermal equilibrium exists between $\mathrm{L}_{2} \mathrm{Mo}_{2}\left(\mu, \eta^{2}-\mathrm{S}_{2}\right)(\mu-\mathrm{S})_{2}(\mathrm{~A})$ and $\operatorname{syn}-\mathrm{L}_{2} \mathrm{Mo}_{2}(\mu$ $S_{2} S_{2}$ (D) which depends strongly on the ligands.
\end{abstract}

\section{Zusammenfassung}

Es wird die Reaktion der verschiedenartig substituierten Cyclopentadienylkomplexe $\mathrm{L}_{2} \mathrm{M}_{2}(\mathrm{CO})_{4}$ mit Schwefel in Abhängigkeit vom Liganden $\mathbf{L}$ (etmcp $=$ Ethyltetramethylcyclopentadienyl, tbcp $=\mathrm{t}$ Butylcyclopentadienyl, ipcp = Isopropylcyclopentadienyl) und dem Metall $\mathrm{M}(\mathrm{Cr}, \mathrm{Mo}, \mathrm{W})$ untersucht. Für $\mathrm{M}=\mathrm{Cr}$ läßt sich als einziges Produkt etmcp ${ }_{2} \mathrm{Cr}_{2} \mathrm{~S}_{5}$ isolieren. Für $\mathrm{M}=\mathrm{Mo}$ bilden sich drei Isomere $A, D, E$ der Zusammensetzung $\mathrm{L}_{2} \mathrm{Mo}_{2} \mathrm{~S}_{4}(\mathrm{~L}=\mathrm{ipcp}$, etmcp) bzw. die Isomere $A$ und $D$ für $\mathrm{L}=$ tbcp. Im System $\mathrm{L}_{2} \mathrm{~W}_{2}(\mathrm{CO})_{4} / \mathrm{S}_{8}$ entstehen nur zwei Isomere $(D, E)$ der Zusammensetzung $\mathrm{L}_{2} \mathrm{~W}_{2} \mathrm{~S}_{4}$ und zusätzlich $\mathrm{L}_{2} \mathrm{~W}_{2}(\mathrm{CO})_{2} \mathrm{~S}_{3}$ für $\mathrm{L}=$ etmcp. Neben der Charakterisierung der Komplexe durch IR-, ${ }^{1} \mathrm{H}$ und ${ }^{95} \mathrm{Mo}-\mathrm{NMR}$-Spektroskopie werden die Strukturen von syn-etmcp ${ }_{2} \mathrm{M}_{2}(\mu-\mathrm{S})_{2} \mathrm{~S}_{2}(\mathrm{M}=\mathrm{Mo}$, W) durch

Correspondence to: Dr. J. Wachter, Institut für Anorganische Chemie der Universität Regensburg Universitätsstraße 31, W-8400 Regensburg, Deutschland. 
Kristallstrukturanalyse bestimmt, wodurch früher getroffene Strukturzuordnungen korrigiert werden. Weiter wird gezeigt, daß zwischen $\mathrm{L}_{2} \mathrm{Mo}_{2}\left(\mu, \eta^{2}-\mathrm{S}_{2}\right)(\mu-S)_{2}(A)$ und syn- $\mathrm{L}_{2} \mathrm{Mo}_{2}(\mu-S)_{2} \mathrm{~S}_{2}$ (D) ein thermisches Gleichgewicht besteht, das stark ligandenabhängig ist.

\section{Einleitung}

Metallorganische Übergangsmetallsulfide der 6. Nebengruppe beinhalten Strukturelemente, die sie als lösliche Modellverbindungen für heterogene Entschwefelungskatalysatoren interessant machen [1]. Tatsächlich haben sich in der Praxis dimere Cyclopentadienylmolybdänsulfide bereits bei zahlreichen Modellreaktionen, wie beispielweise der Hydrierung von Olefinen, der Hydrogenolyse von C-S-, $\mathrm{C}-\mathrm{O}$ - und $\mathrm{C}-\mathrm{N}$-Bindungen [2] oder der katalytischen Reduktion von $\mathrm{SO}_{2}$ [3] bewährt.

Die Darstellung von Komplexen des Typs pmcp ${ }_{2} \mathbf{M}_{2} S_{n}$ (pmcp = Pentamethylcyclopentadienyl; $\mathrm{M}=\mathrm{Cr}$, Mo, W; $n=4,5$ ) beruht auf der Reaktion der Komplexe pmcp ${ }_{2} \mathrm{M}_{2}(\mathrm{CO})_{4}$ mit elementarem Schwefel unter relativ milden Bedingungen [4]. Während für $\mathrm{M}=\mathrm{Cr}$ die ausschließliche Bildung von $\mathrm{pmcp}_{2} \mathrm{Cr}_{2} \mathrm{~S}_{5}$ [5] beobachtet wurde, ist die Produktpalette für $\mathrm{M}=\mathrm{Mo}$, W wesentlich reichhaltiger. Mit $\mathbf{M}=$ Mo entstehen drei (Schema 1: Strukturtypen A, D, E) mit $M=W$ zwei isomere Formen (Strukturtypen D, E) der Zusammensetzung $\operatorname{pmcp}_{2} \mathbf{M}_{2} \mathbf{S}_{4}$, von denen $\mathbf{A}$ und $\mathbf{E}$ durch Röntgenstrukturanalysen abgesichert sind [6,7]. Der erst in dieser Arbeit einwandfrei gesicherte Strukturtyp D wurde in früheren Arbeiten [6] auf der Basis von IR-spektroskopischen Untersuchungen als Isomer B angenommen.

Obwohl bereits seit längerem bekannt ist, daß die Stammverbindung $\left(\mathrm{C}_{5} \mathrm{H}_{5}\right)_{2} \mathrm{Mo}_{2}(\mathrm{CO})_{4}$ mit Schwefel je nach Stöchiometrie entweder eine oligomere Verbindung oder einen Cluster mit $\mathrm{Mo}_{3} \mathrm{~S}$-Kern ergibt [8], ist der Einfluß weiterer Liganden L auf Bildung und Natur der Produkte noch nicht systematisch untersucht worden. Daher sollen nun in diesen Reaktionstyp die folgenden Liganden cingesetzt werden: Ethyltetramethylcyclopentadienyl (etmcp), t-Butylcyclopentadienyl (tbcp), Isopropylcyclopentadienyl (ipcp).

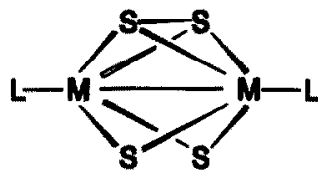

A

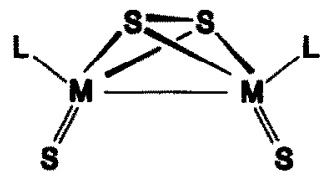

B

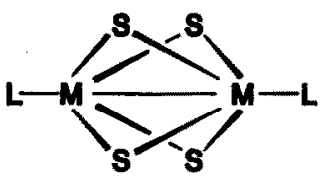

C

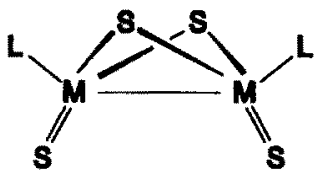

D

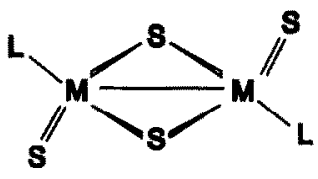

E

Schema 1. Mögliche Isomere für den Komplextyp $\mathrm{L}_{2} \mathrm{Mo}_{2} \mathrm{~S}_{4}$. 
Zunächst wird jedoch die Darstellung und spektroskopische Charakterisierung der Komplexe $\mathrm{L}_{2} \mathrm{M}_{2}(\mathrm{CO})_{4}$ 1-9 beschrieben.

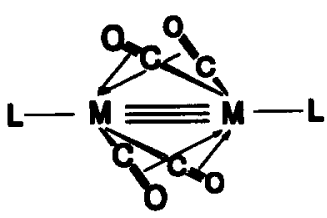

\begin{tabular}{c|ccc|ccc|ccc}
$L$ & \multicolumn{3}{|c|}{ etmcp } & \multicolumn{3}{|c|}{ tocp } & \multicolumn{3}{|c}{ ipcp } \\
\hline$M$ & $C r$ & $M 0$ & $W$ & $C r$ & $M 0$ & $W$ & $C r$ & $M 0$ & $W$ \\
1 & 2 & 3 & 4 & 5 & 6 & 7 & 8 & 9
\end{tabular}

Darstellung und Charakterisierung der Edukte $\mathrm{L}_{2} \mathrm{M}_{2}(\mathrm{CO})_{4} 1-9$ (L=etmcp, tbcp, ipcp; $\mathrm{M}=\mathrm{Cr}, \mathrm{Mo}, \mathrm{W})$

Die Darstellung der Verbindungen $\mathrm{L}_{2} \mathrm{Cr}_{2}(\mathrm{CO})_{4} 4,7$ erfolgte durch Umsetzung von $\left(\mathrm{CH}_{3} \mathrm{CN}\right)_{3} \mathrm{Cr}(\mathrm{CO})_{3}$ mit einer äquimolaren Menge des entsprechenden Liganden in siedendem Toluol. Nach $4 \mathrm{~h}$ wurden nach der chromatographischen Aufarbeitung an silanisiertem Kieselgel grüne Öle erhalten, die erst durch Kristallisation aus Pentan bei $-18^{\circ} \mathrm{C}$ analysenreine Produkte liefern. Die Darstellung der Komplexe 2, 3, 5, 6, 8, 9 konnte dagegen in Anlehnung an die Darstellung von $\operatorname{pmcp}_{2} \mathrm{M}_{2}(\mathrm{CO})_{4}(\mathrm{M}=\mathrm{Mo}, \mathrm{W})$ aus $\mathrm{M}(\mathrm{CO})_{6}(\mathrm{M}=\mathrm{Mo}, \mathrm{W})$ mit äquimolaren Mengen des entsprechenden Liganden durchgeführt werden [9].

Die IR-Spektren der Verbindungen 1-9 (Tab. 1) enthalten jeweils zwei starke Absorptionsbanden asymmetrisch verbrückender Carbonylliganden, wie sie für diesen Verbindungstyp typisch sind [10]. In den ${ }^{1} \mathrm{H}$ - und ${ }^{95} \mathrm{Mo}-\mathrm{NMR}-S p e k t r e n$ von 1-9 (Tab. 1) findet man den Hinweis auf einen symmetrischen Molekülaufbau. Die Aufspaltung der magnetisch inäquivalenten Ringprotonenpaare monosubstituierter Cyclopentadienylliganden in 4-9 ist abhängig vom Zentralmetall: So bestehen ihre Resonanzsignale für $\mathbf{M}=\mathrm{Cr}$ aus zwei pseudo-Tripletts, für $\mathbf{M}=\mathbf{M o}$ dagegen aus lediglich einem Singulett oder für $\mathbf{M}=\mathbf{W}$ aus einem breiten Multiplett. Die Protonen der Methylsubstituenten in den Verbindungen 1-3 sind isochron. Die ${ }^{95}$ Mo-Resonanzen von 2, 5 und 8 liegen im typischen Bereich für die

Tabelle 1

IR-, ${ }^{1} \mathrm{H}-\mathrm{NMR}$ und ${ }^{95}$ Mo-NMR-spektroskopische Daten der Verbindungen 1-9

\begin{tabular}{|c|c|c|c|c|c|c|}
\hline & \multirow{2}{*}{$\begin{array}{l}\text { IR }{ }^{\mathbf{a}} \\
\nu(\mathrm{CO})\end{array}$} & \multicolumn{3}{|l|}{$\delta\left({ }^{1} \mathbf{H}\right)^{b}$} & & \multirow[t]{2}{*}{$\delta\left({ }^{95} \mathrm{Mo}\right)^{c}$} \\
\hline & & $\overline{\mathrm{CH}}$ & $\mathrm{CH}_{2} \mathrm{CH}_{3}{ }^{d}$ & $\mathrm{CH}_{2} \mathrm{CH}_{3}{ }^{d}$ & & \\
\hline$\overline{1}$ & 1880,1855 & $1.82(\mathrm{~s}, 24 \mathrm{H})$ & $2.30(\mathrm{q}, 4 \mathrm{H})$ & $0.87(\mathrm{t}, 6 \mathrm{H})$ & & \\
\hline 2 & 1870,1836 & $1.93(\mathrm{~s}, 24 \mathrm{H})$ & $2.38(\mathrm{q}, 4 \mathrm{H})$ & $0.95(t, 6 \mathrm{H})$ & & $140(260)$ \\
\hline \multirow[t]{2}{*}{3} & 1872,1820 & $2.03(\mathrm{~s}, 24 \mathrm{H})$ & $2.40(\mathrm{q}, 4 \mathrm{H})$ & $0.98(t, 6 H)$ & & \\
\hline & & $\mathrm{C}\left(\mathrm{CH}_{3}\right)_{3}$ & $\mathrm{C}_{5} \mathrm{H}_{4}$ & $\mathrm{CH}\left(\mathrm{CH}_{3}\right)_{2}^{d}$ & $\mathrm{CH}\left(\mathrm{CH}_{3}\right)_{2}{ }^{f}$ & \\
\hline 4 & 1890,1872 & $1.10(\mathrm{~s}, 18 \mathrm{H})$ & $4.57(4 \mathrm{H}), 4.97(4 \mathrm{H})^{e}$ & & & \\
\hline 5 & 1878,1834 & $1.08(\mathrm{~s}, 18 \mathrm{H})$ & $4.74(\mathrm{~s}, 8 \mathrm{H})$ & & & $187(200)$ \\
\hline 6 & 1891,1820 & $1.23(\mathrm{~s}, 18 \mathrm{H})$ & $5.23(\mathrm{~m}, 8 \mathrm{H})$ & & & \\
\hline 7 & 1898,1851 & & $4.45(4 \mathrm{H}), 4.87(4 \mathrm{H})^{e}$ & $1.07(\mathrm{~d}, 12 \mathrm{H})$ & $2.48(\mathrm{~m}, 2 \mathrm{H})$ & \\
\hline 8 & 1876,1838 & & $5.07(\mathrm{~s} ; 8 \mathrm{H})$ & $1.20(\mathrm{~d}, 12 \mathrm{H})$ & $2.79(\mathrm{~m}, 2 \mathrm{H})$ & $173(200)$ \\
\hline 9 & 1888,1832 & & $5.23(\mathrm{~m}, 8 \mathrm{H})$ & $1.15(\mathrm{~d}, 12 \mathrm{H})$ & $2.75(\mathrm{~m}, 2 \mathrm{H})$ & \\
\hline
\end{tabular}

$\bar{a} \mathrm{KBr}\left(\mathrm{cm}^{-1}\right){ }^{b} \mathrm{CDCl}_{3}$-Lösung, i-TMS. ${ }^{c}$ Toluol-Lösung; in Klammern: Linienbreite in $\mathrm{Hz}$. ${ }^{d}{ }^{3} \mathrm{~J}(\mathrm{H}-\mathrm{H})=7 \mathrm{~Hz}$. ${ }^{e}$ pseudo-Tripletts. ${ }^{f}$ Schlecht aufgelöstes Signal. 
Mo-Mo-Dreifachbindung, im Vergleich zu Komplexen des Typs $\mathrm{L}_{2} \mathrm{Mo}_{2}(\mathrm{CO})_{6}$ sind sie um $c a .1800 \mathrm{ppm}$ zu tiefem Feld verschoben [11].

Darstellung der Organometallsulfide 10-19 aus $\mathrm{L}_{2} \mathrm{M}_{2}(\mathrm{CO})_{4}$ 1-9 $(\mathrm{L}=$ etmcp, tbcp, ipcp; $M=C r, M o, W)$ und Schwefel

Setzt man etmcp ${ }_{2} \mathrm{Cr}_{2}(\mathrm{CO})_{4}(1)$ mit $1 / 2 \mathrm{~S}_{8}$ in Toluol $\left(3 \mathrm{~d}, 45^{\circ} \mathrm{C}\right)$ um, so gelangt man zu grünem etmcp $\mathrm{Cr}_{2} \mathrm{~S}_{5}(10)$ in $18 \%$ Ausbeute. $57 \%$ des eingesetzten Edukts können hierbei zurückgewonnen werden. Längere Reaktionszeiten oder Temperaturerhöhung bewirken keine Ausbeutesteigerung.

Läßt man dagegen etmcp ${ }_{2} \mathrm{Mo}_{2}(\mathrm{CO})_{4}(2)$ mit $1 / 2 \mathrm{~S}_{8}$ in Toluol $18 \mathrm{~h}$ bei $45^{\circ} \mathrm{C}$ reagieren, bildet sich neben kleinen Mengen an etmcp ${ }_{2} \mathrm{Mo}_{2}(\mathrm{CO})_{6}$ die Verbindung etmcp ${ }_{2} \mathrm{Mo}_{2} \mathrm{~S}_{4}(11)$ in drei isomeren Formen. Nach chromatographischer Aufarbeitung erhält man blaues etmcp ${ }_{2} \mathrm{Mo}_{2}\left(\mu, \eta^{2}-\mathrm{S}_{2}\right)(\mu-\mathrm{S})_{2}$ (11A), braunes synetmcp ${ }_{2} \mathrm{Mo}_{2}(\mu-S)_{2} \mathrm{~S}_{2}$ (11D) (bisher Strukturtyp B zugeordnet) und rotbraunes anti-etmcp ${ }_{2} \mathrm{Mo}_{2}(\mu-\mathrm{S})_{2} \mathrm{~S}_{2}(11 E)$ im Produktverhältnis 5.4/4.7/1.

Die Reaktion von etmcp $\mathrm{W}_{2}(\mathrm{CO})_{4}(3)$ in Toluol $\left(18 \mathrm{~h}, 45^{\circ} \mathrm{C}\right)$ mit $1 / 2 \mathrm{~S}_{8}$ liefert als Hauptprodukt in 56\% Ausbeute grünes etmcp $\mathrm{W}_{2}(\mathrm{CO})_{2} \mathrm{~S}_{3}(12)$ und als einziges carbonylfreies Produkt grünes syn-etmcp $\mathrm{W}_{2}(\mu-\mathrm{S})_{2} \mathrm{~S}_{2}$ (13D) (bisher Typ B zugeordnet). Bei der weiteren Umsetzung von $12 \mathrm{mit} 1 / 8 \mathrm{~S}_{8}$ unter drastischeren Bedingungen $\left(18 \mathrm{~h}, 100^{\circ} \mathrm{C}\right)$ gelingt die Darstellung der carbonylfreien Isomeren 13D und anti-etmcp ${ }_{2} \mathrm{~W}_{2}(\mu-\mathrm{S})_{2} \mathrm{~S}_{2}(13 \mathrm{E})$ (braun) in guten Ausbeuten.

Während also pmcp- und etmcp ${ }_{2} \mathrm{M}_{2}(\mathrm{CO})_{4}$-Komplexe in etwa gleiche Reaktivität zeigen, reagieren die monosubstituierten Komplexe $\mathrm{L}_{2} \mathrm{M}_{2}(\mathrm{CO})_{4}(\mathrm{~L}=\mathrm{tbcp}$, ipcp) deutlich anders. So lassen sich die $\mathrm{Cr}$-Komplexe 4 und 7 unter Standardbedingungen [5] nicht zu schwefelhaltigen Komplexen umsetzen. Bei $100^{\circ} \mathrm{C}$ erhöht sich lediglich der Anteil an Zersetzungsprodukten.

Die Reaktion der Mo-Komplexe 5 und 8 mit Schwefel wird von der Stöchiometrie beeinflußt: Bei einem Verhältnis Mo/S $=1 / 1$ bilden sich in rascher Reaktion $\left(4 \mathrm{~h}, 45^{\circ} \mathrm{C}\right)$ in Toluol die rotvioletten Verbindungen $\mathrm{L}_{4} \mathrm{Mo}_{4} \mathrm{~S}_{4}(\mathrm{~L}=$ tbcp 14: $62 \%$, $\mathrm{L}=$ ipcp 15: $56 \%$ ). Erhöht man den Schwefelanteil auf ein Verhältnis von $\mathrm{Mo} / \mathrm{S}$ $=1 / 2$, so erhält man nach $7 \mathrm{~h}$ unter sonst analogen Bedingungen zwei $(\mathrm{L}=\mathrm{tbcp})$ bzw. drei Isomere $(\mathrm{L}=\mathrm{ipcp})$ der Zusammensetzung $\mathrm{L}_{2} \mathrm{Mo}_{2} \mathrm{~S}_{4}$. Während die Isolierung und Charakterisierung von rotbraunem anti-tbcp ${ }_{2} \mathrm{Mo}_{2}\left(\mu-\mathrm{S}_{2} \mathrm{~S}_{2}(16 \mathrm{E})\right.$, braunem syn-ipcp ${ }_{2} \mathrm{Mo}_{2}(\mu-\mathrm{S})_{2} \mathrm{~S}_{2}$ (17D) und rotbraunem anti-ipcp ${ }_{2} \mathrm{Mo}_{2}(\mu-\mathrm{S})_{2} \mathrm{~S}_{2}$ (17E) unproblematisch verläuft, lassen sich im Zuge der chromatographischen Reinigung Eluate mit der für 16A und 17A erwarteten typischen blauen Farbe beobachten. Nach Abziehen des Lösungsmittels erhält man jedoch braune, unlösliche Feststoffe. Ein solches Verhalten wurde an unabhängig dargestelltem $\operatorname{mecp}_{2} \mathrm{Mo}_{2}\left(\mu, \eta^{2}-\mathrm{S}_{2}\right)(\mu-\mathrm{S})_{2}$ (mecp = Methylcyclopentadienyl) beobachtet [12], jedoch nicht näher untersucht. Hierbei dürfte es sich um eine konzentrationsabhängige Oligomerisierung von 16A, 17A handeln $\left[13^{*}\right]$.

Erhöht man den Schwefelanteil noch weiter, so erhält man ein tiefrotes Öl, das jedoch nicht genügend gereinigt werden konnte. Sein Massenspektrum deutet auf

* Die Literaturnummer mit einem Sternchen deuten eine Bemerkung in der Literaturliste an. 
die Zusammensetzung $\mathrm{L}_{2} \mathrm{Mo}_{2} \mathrm{~S}_{4}(\mathrm{~L}=$ tbcp, ipcp) hin, im IR-Spektrum fehlen die Streckschwingungen terminaler Schwefelliganden.

Die Umsetzung von $\mathrm{L}_{2} \mathrm{~W}_{2}(\mathrm{CO})_{4} 6,9$ mit $1 / 2 \mathrm{~S}_{8}$ bei $45^{\circ} \mathrm{C}$ in Toluol liefert nur die carbonylfreien Produkte anti- $\mathrm{L}_{2} \mathrm{~W}_{2}(\mu-\mathrm{S})_{2} \mathrm{~S}_{2}(\mathrm{~L}=$ tbcp 18E: 22\%; ipcp 19E: $18 \%)$ und syn $-\mathrm{L}_{2} \mathrm{~W}_{2}(\mu-\mathrm{S})_{2} \mathrm{~S}_{2}(\mathrm{~L}=$ tbcp 18D: 28\%; ipcp 19D: 14\%). Die Isomeren können wie in allen vorhergehenden Fällen durch Säulenchromatographie getrennt werden.

\section{Spektroskopische und strukturelle Charakterisierung der Verbindungen 10-19}

Struktur von etmcp ${ }_{2} \mathrm{Cr}_{2} \mathrm{~S}_{5}$ (10)

Die Struktur von etmcp $\mathrm{Cr}_{2} \mathrm{~S}_{5}(\mathbf{1 0})$ ist nicht nur aufgrund einer Farbanalogie, sondern auch sehr ähnlicher IR- und ${ }^{1}$ H-NMR-Daten (Tab. 2, 3) mit dem für pmcp $\mathrm{Cr}_{2} \mathrm{~S}_{5}$ durch Röntgenstrukturanalyse nachgewiesenen Strukturtyp [5] vereinbar. D.h., 10 ist der bisher zweite Komplex mit einer $\eta^{1}$-S,S-Brücke.

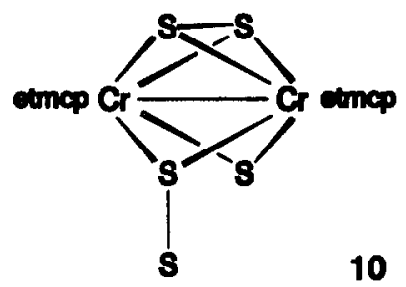

Röntgenstrukturanalyse von syn-etmcp ${ }_{2} \mathrm{Mo}_{2}(\mu-S)_{2} S_{2}$ (11D)

Da eine einwandfrei Unterscheidung zwischen den für die Mo- und W-Komplexe relevanten Isomeren $\mathbf{B}$ und $\mathbf{D}$ bisher unmöglich gewesen war, wurde an einem aus Toluol bei $-18^{\circ} \mathrm{C}$ gezogenem Einkristall von 11D eine Röntgenstrukturanalyse durchgeführt (Fig. 1, Tab. 4, 5). Die Struktur von $11 D$ läßt

Tabelle 2

IR-spektroskopische Daten der Komplexe 10-19 $\left(\mathrm{KBr}, \mathrm{cm}^{-1}\right)$

\begin{tabular}{|c|c|c|c|}
\hline & $\nu(\mathrm{CO})$ & $\nu(\mathrm{M}=\mathrm{S})$ & $\nu(\mathbf{S}-\mathbf{S}, \mathbf{M}-\mathbf{S}-\mathbf{M})$ \\
\hline 10 & & & $500 w, 445 w$ \\
\hline $11 \mathrm{~A}$ & & & $482 \mathrm{vw}, 385 \mathrm{~m}, 312 \mathrm{vw}$ \\
\hline 11D & & $485 \mathrm{~s}, 474 \mathrm{sh}$ & $450 \mathrm{w}, 370 \mathrm{~m}, 340 \mathrm{vw}$ \\
\hline 11E & & $500 \mathrm{~s}$ & $461 \mathrm{~m}, 440 \mathrm{~m}, 382 \mathrm{~m}, 331 \mathrm{~m}$ \\
\hline 12 & $1932 \mathrm{vs}, 1852 \mathrm{vs}$ & $482 \mathrm{~s}$ & \\
\hline 13D & & $487 \mathrm{~s}, 475 \mathrm{sh}, 465 \mathrm{sh}$ & $438 \mathrm{~m}, 425 \mathrm{w}, 367 \mathrm{w}, 330 \mathrm{w}$ \\
\hline 13E & & $492 \mathrm{~s}, 468 \mathrm{~s}$ & $428 \mathrm{~m}, 374 \mathrm{w}, 330 \mathrm{ww}$ \\
\hline 14 & & & $461 w$ \\
\hline 15 & & & $442 w, 360 w, 330 w$ \\
\hline $16 E$ & & $486 \mathrm{~s}$ & $437 m, 335 w$ \\
\hline 17D & & 493s, 484sh & $438 \mathrm{~m}, 348 w$ \\
\hline $17 \mathbf{E}$ & & $487 \mathrm{~s}$ & $443 \mathrm{~m}, 365 \mathrm{w}, 350 \mathrm{w}$ \\
\hline 18D & & $494 \mathrm{~s}, 487 \mathrm{sh}$ & $462 m, 431 m, 429 m$ \\
\hline $18 \mathbf{E}$ & & $488 \mathrm{~s}$ & $439 \mathrm{~m}, 342 \mathrm{w}$ \\
\hline 19D & & 493s, 484sh & $438 m, 348 w$ \\
\hline $19 E$ & & $487 \mathrm{~s}$ & $443 \mathrm{~m}, 365 \mathrm{w}, 350 \mathrm{w}$ \\
\hline
\end{tabular}


Tabelle 3

NMR-spektroskopische Daten der Verbindungen 10-19

\begin{tabular}{|c|c|c|c|c|c|}
\hline & $\delta\left({ }^{1} \mathrm{H}\right)^{a}$ & & & & $\delta\left(^{95} \mathrm{Mo}\right)^{h}$ \\
\hline & $\mathrm{CH}_{3}$ & $\mathrm{CH}_{2} \mathrm{CH}_{3}{ }^{c}$ & $\mathrm{CH}_{2} \mathrm{CH}_{3}{ }^{c}$ & & \\
\hline 10 & $2.17(\mathrm{~s}, 24 \mathrm{H})$ & $2.50(q, 4 \mathrm{H})$ & $1.27(\mathrm{t}, 6 \mathrm{H})$ & & \\
\hline $11 \mathrm{~A}$ & $2.25(\mathrm{~s}, 24 \mathrm{H})$ & $2.53(q, 4 \mathrm{H})$ & $1.17(\mathrm{t}, 6 \mathrm{H})$ & & $426(130)$ \\
\hline 11D & $2.03(\mathrm{~s}, 12 \mathrm{H}), 2.08(\mathrm{~s}, 12 \mathrm{H})$ & $2.42(\mathrm{q}, 4 \mathrm{H})$ & $1.15(\mathrm{t}, 6 \mathrm{H})$ & & $737(200)$ \\
\hline 11E & $2.10(s, 24 \mathrm{H})$ & $2.45(\mathrm{q}, 4 \mathrm{H})$ & $1.14(\mathrm{t}, 6 \mathrm{H})$ & & $458(350)$ \\
\hline 12 & $\begin{array}{l}1.83(\mathrm{~s}, 6 \mathrm{H}), 2.10(\mathrm{~s}, 6 \mathrm{H}) \\
2.15(\mathrm{~s}, 6 \mathrm{H}), 2.18(\mathrm{~s}, 6 \mathrm{H})\end{array}$ & $2.63(\mathrm{~m}, 4 \mathrm{H})$ & $1.07(t, 6 \mathrm{H})$ & & \\
\hline 13D & $2.23(\mathrm{~s}, 24 \mathrm{H})$ & $2.50(\mathrm{q}, 4 \mathrm{H})$ & $1.12(\mathrm{t}, 6 \mathrm{H})$ & & \\
\hline 13E & $2.18(\mathrm{~s}, 12 \mathrm{H}), 2.30(\mathrm{~s}, 12 \mathrm{H})$ & $2.75(q, 4 H)$ & $1.10(\mathrm{t}, 6 \mathrm{H})$ & & \\
\hline & $\mathrm{C}\left(\mathrm{CH}_{3}\right)_{3}$ & $\mathrm{C}_{5} \mathrm{H}_{4}$ & $\left.\mathrm{CH}(\mathrm{CH})_{3}\right)^{c}$ & $\mathrm{CH}\left(\mathrm{CH}_{3}\right)_{2} f$ & \\
\hline 14 & $1.12(\mathrm{~s}, 36 \mathrm{H})$ & $4.90(8 \mathrm{H}), 5.40(8 \mathrm{H})^{d}$ & & & \\
\hline 15 & & $5.53(16 \mathrm{H})^{e}$ & $1.10(\mathrm{~d}, 24 \mathrm{H})$ & $2.95(\mathrm{~m}, 4 \mathrm{H})$ & \\
\hline $16 E$ & $1.73(\mathrm{~s}, 18 \mathrm{H})$ & $5.13(4 \mathrm{H}), 5.98(4 \mathrm{H})^{d}$ & & & \\
\hline 17A & & & & & $116(160)^{g}$ \\
\hline 17D & & $5.23(\mathrm{~s}, 4 \mathrm{H}), 5.85(4 \mathrm{H})^{d}$ & $1.50(\mathrm{~d}, 12 \mathrm{H})$ & $3.18(\mathrm{~m}, 2 \mathrm{H})$ & $387(130)$ \\
\hline $17 E$ & & $5.70(\mathrm{~s}, 8 \mathrm{H})$ & $1.43(\mathrm{~d}, 12 \mathrm{H})$ & $3.03(\mathrm{~m}, 2 \mathrm{H})$ & $230(420)$ \\
\hline 18D & $1.77(\mathrm{~s}, 18 \mathrm{H})$ & $4.83(4 \mathrm{H}), 6.03(4 \mathrm{H})^{d}$ & & & \\
\hline 18E & $1.76(\mathrm{~s}, 18 \mathrm{H})$ & $5.26(4 \mathrm{H}), 6.02(4 \mathrm{H})^{d}$ & & & \\
\hline 19D & & $5.23(4 \mathrm{H}), 5.80(4 \mathrm{H})^{d}$ & $1.53(\mathrm{~d}, 12 \mathrm{H})$ & $3.33(\mathrm{~m}, 2 \mathrm{H})$ & \\
\hline $19 \mathrm{E}$ & & $5.77(\mathrm{~s}, 8 \mathrm{H})$ & $1.43(\mathrm{~d}, 12 \mathrm{H})$ & $3.08(\mathrm{~m}, 2 \mathrm{H})$ & \\
\hline
\end{tabular}

sich durch je ein Paar cis-ständiger Monosulfidbrücken und terminaler Schwefelliganden beschreiben. Der gleiche Ligandensatz liegt centrosymmetrisch angeordnet in anti-pmcp ${ }_{2} \mathrm{Mo}_{2}(\mu-\mathrm{S})_{2} \mathrm{~S}_{2}$ vor [7], was nahezu gleiche Mo-Mo-Abstände in

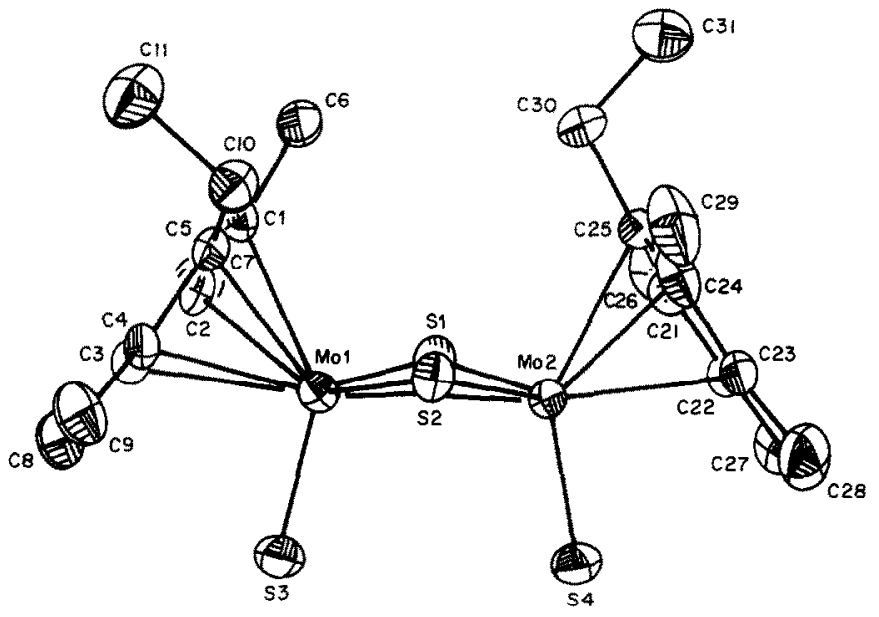

Fig. 1. Molekülstruktur von etmcp $\mathrm{Mo}_{2} \mathrm{~S}_{4}$ (11D) (oRTEP). 
Tabelle 4

Atomkoordinaten $\left(\times 10^{4}\right)$ und thermische Parameter $\left(U_{e q} \times 10^{3}\right)$ von etmcp ${ }_{2} \mathrm{Mo}_{2} \mathrm{~S}_{4}(11 D)$

\begin{tabular}{lrrrl}
\hline Atom & \multicolumn{1}{l}{$\boldsymbol{x}$} & \multicolumn{1}{l}{$\boldsymbol{z}$} & $U_{\text {eq }}$ \\
\hline Mo(1) & $1715(1)$ & $1629(1)$ & $974(1)$ & $28(1)$ \\
Mo(2) & $3431(1)$ & $2049(1)$ & $1673(1)$ & $29(1)$ \\
S(1) & $1877(1)$ & $1034(1)$ & $1674(1)$ & $38(1)$ \\
S(2) & $3619(1)$ & $2266(1)$ & $919(1)$ & $34(1)$ \\
S(3) & $419(2)$ & $2666(1)$ & $1037(1)$ & $47(1)$ \\
$S(4)$ & $2665(2)$ & $3232(1)$ & $1922(1)$ & $47(1)$ \\
$C(1)$ & $1968(5)$ & $252(4)$ & $624(2)$ & $33(2)$ \\
$C(2)$ & $703(5)$ & $357(4)$ & $716(2)$ & $36(2)$ \\
C(3) & $255(5)$ & $1034(4)$ & $443(2)$ & $37(2)$ \\
$C(4)$ & $1253(5)$ & $1378(4)$ & $208(2)$ & $32(2)$ \\
$C(5)$ & $2307(5)$ & $878(4)$ & $310(2)$ & $30(2)$ \\
$C(6)$ & $2759(6)$ & $-471(4)$ & $792(2)$ & $51(2)$ \\
$C(7)$ & $-41(6)$ & $-229(4)$ & $1008(2)$ & $58(3)$ \\
$C(8)$ & $-1060(6)$ & $1298(5)$ & $389(2)$ & $56(3)$ \\
$C(9)$ & $1187(6)$ & $2107(4)$ & $-117(2)$ & $55(3)$ \\
$C(10)$ & $3526(5)$ & $962(4)$ & $79(2)$ & $43(2)$ \\
$C(11)$ & $3560(6)$ & $450(5)$ & $-349(2)$ & $68(3)$ \\
$C(21)$ & $4443(6)$ & $1159(4)$ & $2209(2)$ & $40(2)$ \\
$C(22)$ & $4675(6)$ & $2025(4)$ & $2338(2)$ & $41(2)$ \\
$C(23)$ & $5374(6)$ & $2437(4)$ & $2008(2)$ & $41(2)$ \\
$C(24)$ & $5594(5)$ & $1799(5)$ & $1671(2)$ & $44(2)$ \\
$C(25)$ & $5025(6)$ & $1020(4)$ & $1797(2)$ & $40(2)$ \\
$C(26)$ & $3832(7)$ & $486(5)$ & $2490(3)$ & $78(3)$ \\
$C(27)$ & $4327(7)$ & $2439(5)$ & $2767(2)$ & $76(3)$ \\
$C(28)$ & $5838(7)$ & $3352(4)$ & $2021(3)$ & $74(3)$ \\
$C(29)$ & $6430(6)$ & $1909(6)$ & $1277(2)$ & $77(3)$ \\
$C(30)$ & $5137(7)$ & $171(5)$ & $1564(3)$ & $71(3)$ \\
$C(31)$ & $6292(7)$ & $-303(5)$ & $1694(3)$ & $93(4)$ \\
\hline & & & &
\end{tabular}

Tabelle 5

Bindungslängen $(\AA ̊)$ und -winkel $\left(^{\circ}\right)$ von $11 \mathrm{D}$

\begin{tabular}{|c|c|c|c|}
\hline $\mathrm{Mo}(1)-\mathrm{Mo}(2)$ & $2.895(1)$ & Mo(2)-S(1) & $2.311(2)$ \\
\hline $\operatorname{Mo}(1)-S(1)$ & $2.312(2)$ & $\mathrm{Mo}(2)-\mathrm{S}(2)$ & $2.310(2)$ \\
\hline $\mathrm{Mo}(1)-\mathrm{S}(2)$ & $2.306(2)$ & $\mathrm{Mo}(2)-\mathrm{S}(4)$ & $2.146(2)$ \\
\hline $\operatorname{Mo}(1)-S(3)$ & $2.148(2)$ & $M o(2)-C(21)$ & $2.394(6)$ \\
\hline $\operatorname{Mo}(1)-C(1)$ & $2.393(6)$ & $\operatorname{Mo}(2)-C(22)$ & $2.424(6)$ \\
\hline $\mathrm{Mo}(1)-\mathrm{C}(2)$ & $2.386(6)$ & $M o(2)-C(23)$ & $2.426(6)$ \\
\hline $\mathrm{Mo}(1)-\mathrm{C}(3)$ & $2.441(6)$ & $\mathrm{Mo}(2)-\mathrm{C}(24)$ & $2.393(6)$ \\
\hline $\mathrm{Mo}(1)-\mathrm{C}(4)$ & $2.399(6)$ & $\mathrm{Mo}(2)-\mathrm{C}(25)$ & $2.387(6)$ \\
\hline $\operatorname{Mo}(1)-C(5)$ & $2.404(5)$ & & \\
\hline $\mathrm{Mo}(2)-\mathrm{Mo}(1)-\mathrm{S}(1)$ & $51.2(1)$ & $\mathrm{Mo}(1)-\mathrm{Mo}(2)-\mathrm{S}(2)$ & $51.1(1)$ \\
\hline$M o(2)-M o(1)-S(2)$ & $51.2(1)$ & $M o(1)-M o(2)-S(1)$ & $51.2(1)$ \\
\hline$S(1)-M o(1)-S(2)$ & $99.6(1)$ & $S(1)-M o(2)-S(2)$ & 99.5(1) \\
\hline$M o(2)-M o(1)-S(3)$ & 101.2(1) & $\mathrm{Mo}(1)-\mathrm{Mo}(2)-\mathrm{S}(4)$ & $101.2(1)$ \\
\hline$S(1)-M o(1)-S(3)$ & $105.5(1)$ & $S(1)-M o(2)-S(4)$ & $107.0(1)$ \\
\hline S(2)-Mo(1)-S(3) & $106.3(1)$ & S(2)-Mo(2)-S(4) & 104.8(1) \\
\hline$M o(1)-S(1)-M o(2)$ & $77.6(1)$ & & \\
\hline $\operatorname{Mo}(1)-S(2)-\operatorname{Mo}(2)$ & $77.7(1)$ & & \\
\hline
\end{tabular}



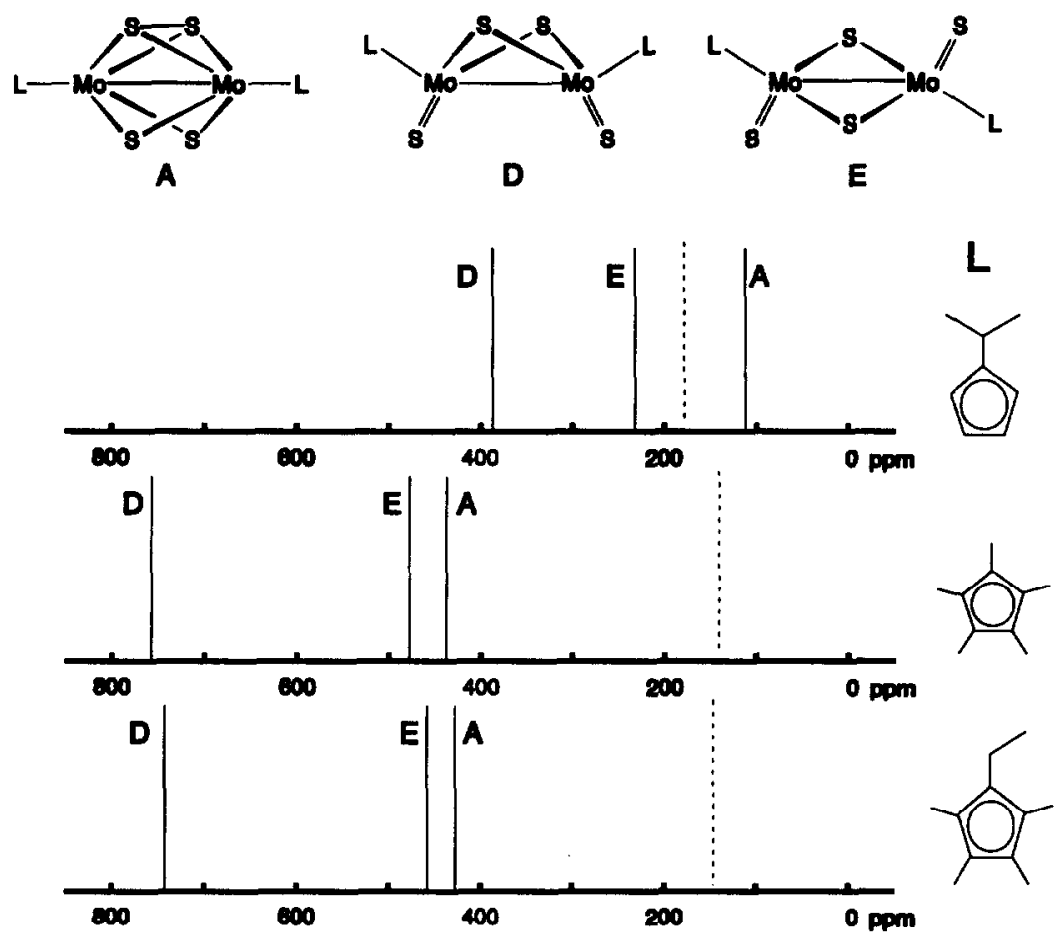

Fig. 2. Vergleich der ${ }^{95} \mathrm{Mo}-\mathrm{NMR}-S p e k t r e n$ von $\mathrm{L}_{2} \mathrm{Mo}_{2} \mathrm{~S}_{4}$-Isomeren $(\mathrm{L}=$ pmcp [11], $\mathrm{L}=$ etmcp: 11, $\mathrm{L}=$ ipcp: 17) und $\mathrm{L}_{2} \mathrm{Mo}_{2}(\mathrm{CO})_{4}(\ldots-\ldots)$.

beiden Komplexen zur Folge hat. Zwischen den zueinander gefalteten Brückenschwefelatomen (Diederwinkel $157.0^{\circ}$ ) besteht keinerlei bindende Wechselwirkung $(d(\mathrm{~S}(1) \cdots \mathrm{S}(2))=3.527 \AA)$. Das Strukturelement syn-ständiger Monosulfidbrücken wurde bereits in dem Halboxokomplex $\operatorname{mecp}_{2} \mathrm{Mo}_{2}(\mu-\mathrm{S})_{2} \mathrm{O}_{2}$ gefunden [15], dessen $\mathrm{Mo}_{2} \mathrm{~S}_{2}$-Gerüst ähnliche Strukturparameter wie das von 11D aufweist.

Strukturen der Mo-Komplexe 14-17

Von dem auf anderem Weg zugänglichen ipcp ${ }_{4} \mathrm{Mo}_{4} \mathrm{~S}_{4}(15)$ existiert bereits eine Röntgcnstrukturanalyse [16]. Sein cubanartig aufgebautes Molybdänsulfidgerüst mit $6 \mathrm{M}$-M-Bindungen dürfte sich auch in tbcp ${ }_{4} \mathrm{Mo}_{4} \mathrm{~S}_{4}(14)$ wiederfinden, wofür neben der Farbanalogie die IR- (lediglich schwache $\mathrm{M}-\mathrm{S}-\mathrm{M}$-Schwingungen im Bereich unter $600 \mathrm{~cm}^{-1}$ ) und ${ }^{1} \mathrm{H}-\mathrm{NMR}$-Spektren (symmetrischer Molekülaufbau) sprechen (Tab. 2, 3).

Ausgehend von der nun zweifelsfrei bewiesenen Präsenz zweier Monosulfidbrücken in 11D lassen sich mit Hilfe der ${ }^{95} \mathrm{Mo}$-NMR-Spektroskopie nunmehr einwandfreie Strukturkorrelationen durchführen. Die $\delta($ Mo)-Werte von 11A, D, E (Tab. 3) und der analogen pmcp-Komplexe [11] stimmen überein und beweisen damit die strukturelle Identität beider Systeme. Die $\delta(\mathrm{Mo})$-Werte der ipcpVerbindungen sind dagegen um $c a$. 220-350 ppm nach hohem Feld verschoben, jedoch ist hier der gleiche Gang $\delta(\mathrm{Mo}) \mathbf{D}>\delta(\mathrm{Mo}) \mathrm{A}>\delta(\mathrm{Mo}) \mathrm{E}$ wie bei den pmcp-Verbindungen anzutreffen (Fig. 2). Damit, unter Einbeziehung der Farb- 
Tabelle 6

Atomkoordinaten $\left(\times 10^{4}\right)$ und thermische Parameter $\left(U_{\mathrm{eq}} \times 10^{3}\right)$ von etmcp $2 \mathrm{~W}_{2} \mathrm{~S}_{4}(13 \mathrm{D})$

\begin{tabular}{|c|c|c|c|c|}
\hline Atom & $x$ & $y$ & $z$ & $U_{\mathrm{cq}}$ \\
\hline$\overline{W(1)}$ & $1703(1)$ & $1637(1)$ & $974(1)$ & $28(1)$ \\
\hline$W(2)$ & $3421(1)$ & $2059(1)$ & $1673(1)$ & $29(1)$ \\
\hline$S(1)$ & $1847(2)$ & $1057(2)$ & $1678(1)$ & $38(1)$ \\
\hline $\mathbf{S}(2)$ & $3602(2)$ & $2283(2)$ & $917(1)$ & $35(1)$ \\
\hline$S(3)$ & $389(3)$ & $2667(2)$ & $1030(1)$ & $47(1)$ \\
\hline$S(4)$ & $2662(3)$ & $3247(2)$ & $1925(1)$ & $47(1)$ \\
\hline$C(1)$ & $2302(9)$ & $873(6)$ & $309(3)$ & 31(3) \\
\hline$C(2)$ & 1981(9) & $253(7)$ & $626(3)$ & $35(3)$ \\
\hline$C(3)$ & $701(9)$ & $359(7)$ & $723(3)$ & $37(3)$ \\
\hline$C(4)$ & $242(10)$ & $1026(7)$ & $449(4)$ & $42(4)$ \\
\hline$C(5)$ & $1292(9)$ & $1376(7)$ & $206(3)$ & $34(3)$ \\
\hline$C(6)$ & $3513(9)$ & $973(7)$ & $79(3)$ & 44(4) \\
\hline$C(7)$ & $2759(11)$ & $-457(7)$ & $802(4)$ & $53(4)$ \\
\hline$C(8)$ & $-40(11)$ & $-220(8)$ & $1020(4)$ & $60(5)$ \\
\hline$C(9)$ & $-1081(9)$ & $1283(8)$ & $397(4)$ & $53(4)$ \\
\hline$C(10)$ & $1205(11)$ & $2091(8)$ & $-133(4)$ & $62(5)$ \\
\hline$C(11)$ & $3564(10)$ & $438(9)$ & $-347(4)$ & $66(5)$ \\
\hline$C(12)$ & $5015(11)$ & $1031(7)$ & $1792(3)$ & $40(4)$ \\
\hline$C(13)$ & $4421(10)$ & $1161(8)$ & $2201(3)$ & 41(4) \\
\hline$C(14)$ & $4668(11)$ & $2037(8)$ & $2339(3)$ & $48(4)$ \\
\hline$C(15)$ & $5381(10)$ & $2444(7)$ & 1997(3) & $38(3)$ \\
\hline$C(16)$ & $5588(9)$ & $1811(8)$ & $1664(3)$ & $46(4)$ \\
\hline$C(17)$ & $5130(11)$ & $183(8)$ & $1551(4)$ & $66(5)$ \\
\hline$C(18)$ & $3810(12)$ & $503(8)$ & $2489(5)$ & $83(6)$ \\
\hline$C(19)$ & $4298(12)$ & $2430(10)$ & $2778(3)$ & $78(6)$ \\
\hline$C(20)$ & $5854(12)$ & $3340(8)$ & $2019(5)$ & $72(5)$ \\
\hline$C(21)$ & $6426(10)$ & $1929(10)$ & $1277(4)$ & $76(6)$ \\
\hline$C(22)$ & $6293(12)$ & $-305(9)$ & $1684(5)$ & $96(7)$ \\
\hline
\end{tabular}

analogie und aufgrund der IR-Absorptionen im Bereich unter $600 \mathrm{~cm}^{-1}$ sollten die ipcp-Isomere 17D, E eine zu den etmcp- bzw. pmcp-Verbindungen analoge Molekülstruktur aufweisen. Für die tbcp-Komplexe 16 konnten keine ${ }^{95}$ Mo-NMRSpektren aufgenommen werden. Im Gegensatz zu dem in $\mathrm{L}_{2} \mathrm{Mo}_{2}(\mathrm{CO})_{4}$ festgestellten Ligandeneinfluß (Fig. 2) bewirkt der niedriger substituierte ipcp-Ligand eine wesentlich stärkere Abschirmung der Mo-Kerne. Dieser Trend scheint auch in anderen Cyclopentadienyl-Mo-sulfiden eine Rolle zu spielen [11,17,18].

Röntgenstrukturanalyse von syn-etmcp ${ }_{2} \mathrm{~W}_{2} \mathrm{~S}_{4}$ (13D); Strukturen der Komplexe 12, 18 und 19

Die wie 11D ebenfalls kontrovers diskutierte Struktur von 13D konnte durch eine an einem aus Toluol bei $-18^{\circ} \mathrm{C}$ gezogenen Einkristall durchgeführte Röntgenstrukturanalyse aufgeklärt werden (Tab. 6, 7). Sie ist nahezu identisch mit der von syn-11D. Das heißt, das Metallsulfidgerüst ist auch hier durch zwei terminale Schwefelliganden (mittlerer Abstand W=S $2.153 \AA$ ) und zwei synständige Monosulfidbrïcken aufgebaut (Fig. 3). Der Winkel zwischen den Flächen $\mathrm{W}(1)-\mathrm{S}(1)-\mathrm{W}(2)$ und $\mathrm{W}(1)-\mathrm{S}(2)-\mathrm{W}(2)$ beträgt $158.5^{\circ}$. Zwei syn-ständige Monosulfidbrücken liegen auch in $\mathrm{Cp}_{2} \mathrm{~W}_{2} \mathrm{~S}_{2}\left(\mathrm{SC}_{2} \mathrm{H}_{2} \mathrm{~S}\right)_{2}$ vor, hier beträgt dieser Winkel $159.2^{\circ}$ [19]. Der Wolfram-Wolfram-Abstand (2.891 $\left.\mathrm{A}\right)$ ist typisch für schwefelverbrückte $\mathrm{W}^{\mathrm{V}}$-Verbindungen [20-22]. 
Tabelle 7

Bindungslängen $(\AA)$ und -winkel $\left(^{\circ}\right)$ von $13 D$

\begin{tabular}{lcll}
\hline W(1)-W(2) & $2.891(1)$ & & \\
W(1)-S(1) & $2.307(3)$ & W(2)-S(1) & $2.314(3)$ \\
W(1)-S(2) & $2.306(3)$ & W(2)-S(2) & $2.308(2)$ \\
W(1)-S(3) & $2.152(3)$ & W(2)-S(4) & $2.155(3)$ \\
W(1)-C(1) & $2.414(9)$ & W(2)-C(12) & $2.385(11)$ \\
W(1)-C(2) & $2.403(10)$ & W(2)-C(13) & $2.379(11)$ \\
W(1)-C(3) & $2.383(10)$ & W(2)-C(14) & $2.423(11)$ \\
W(1)-C(4) & $2.435(11)$ & W(2)-C(15) & $2.424(10)$ \\
W(1)-C(5) & $2.386(10)$ & W(2)-C(16) & $2.395(10)$ \\
W(2)-W(1)-S(1) & $51.4(1)$ & W(1)-W(2)-S(1) & $51.2(1)$ \\
W(2)-W(1)-S(2) & $51.2(1)$ & W(1)-W(2)-S(2) & $51.2(1)$ \\
S(1)-W(1)-S(2) & $100.1(1)$ & $S(1)-W(2)-S(2)$ & $99.9(1)$ \\
W(2)-W(1)-S(3) & $101.9(1)$ & W(1)-W(2)-S(4) & $101.6(1)$ \\
S(1)-W(1)-S(3) & $105.2(1)$ & $S(1)-W(2)-S(4)$ & $106.6(1)$ \\
S(2)-W(1)-S(3) & $106.4(1)$ & $S(2)-W(2)-S(4)$ & $104.6(1)$ \\
W(1)-S(1)-W(2) & $77.4(1)$ & & \\
W(1)-S(2)-W(2) & $77.6(1)$ & & \\
\hline
\end{tabular}

Mit der nun eindeutigen Strukturzuordnung von 13D können aufgrund der spektroskopischen Daten (Tab. 2, 3) und der Farbanalogie "grün" syn- $\mathrm{L}_{2} \mathbf{W}_{2}(\mu$ $S)_{2} S_{2}(L=$ tbcp 18D, ipcp 19D) als gesichert gelten. Die braunen Isomeren $\mathbf{E}$ von 13, 18 und 19 besitzen laut IR-Spektren terminal koordinierte Schwefelliganden, so daß sie wie die analogen Molybdän-Komplexe anti-Struktur aufweisen sollten.

Widersprüchlich sind die ${ }^{1} \mathrm{H}-\mathrm{NMR}-$ Spektren in Bezug auf die Aufspaltung der Resonanzen der Ringprotonen in monosubstituierten Komplexen 17-19. Dies gilt auch für die unterschiedliche Aufspaltung der Cp-Methylresonanzen durch die Ethylgruppen in den Komplexen $11 D$ und $13 D$ und ist letztlich wohl als Resultat

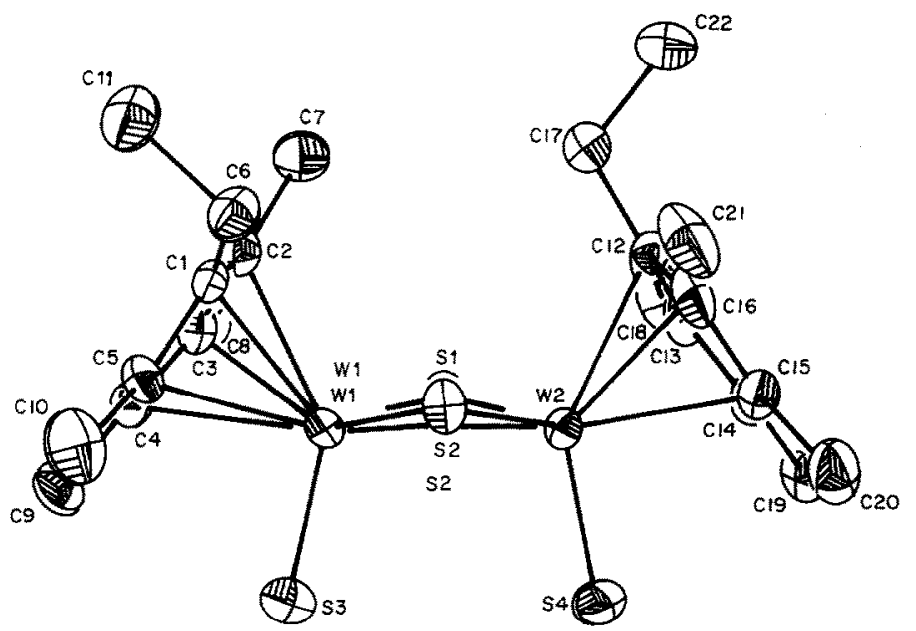

Fig. 3. Molekülstruktur von etmcp ${ }_{2} \mathrm{~W}_{2} \mathrm{~S}_{4}$ 13D (ORTEP). 
des elektronischen und sterischen Einflusses des $\mathbf{M}_{2} \mathbf{S}_{4}$-Kerns auf die Cp-Liganden zu verstehen.

Eine Sonderstellung nimmt 12 ein, das laut IR-spektroskopischen Daten terminale $\mathrm{CO}$ - und Schwefel-Liganden enthält. Als Folge resultieren wie in pmpc $_{2} \mathrm{~W}_{2}(\mathrm{CO})_{2} \mathrm{~S}_{3}$, dessen Struktur bereits durch Röntgenstrukturanalyse aufgeklärt wurde [6], zwei unterschiedliche W-Zentren, was sich in einer Verdoppelung der möglichen $\mathrm{CH}_{3}$-Resonanzen auf vier (Tab. 3) ausdrückt.

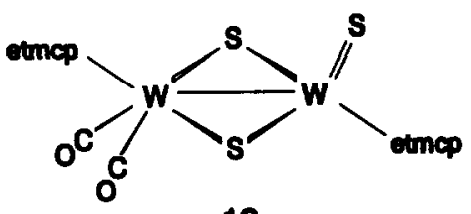

12

Isomerisierungsreaktionen von $\mathrm{L}_{2} \mathrm{Mo}_{2}\left(\mu, \eta^{2}-S_{2}\right)(\mu-S)_{2}(L=$ etmcp, tbcp, ipcp)

Eine besondere Eigenschaft von pmcp ${ }_{2} \mathrm{Mo}_{2}\left(\mu, \eta^{2}-\mathrm{S}_{2}\right)(\mu-\mathrm{S})_{2}$ ist die Fähigkeit, sich durch Erwärmen seiner Lösungen in die beiden anderen Strukturisomere umzuwandeln [6]. Wir fanden nunmehr, daß diese Isomerisierungsreaktionen durch Ligandenvariation beeinflußt werden können. In einem ${ }^{1} \mathrm{H}$-NMR-Experiment isomerisiert etmcp ${ }_{2} \mathrm{Mo}_{2}\left(\mu, \eta^{2}-\mathrm{S}_{2}\right)(\mu-\mathrm{S})_{2}(11 \mathrm{~A})$ in $\mathrm{CDCl}_{3}$-Lösung bei $50^{\circ} \mathrm{C} \mathrm{zu}$ syn-11D. Nach $24 \mathrm{~h}$ stellt sich ein Gleichgewicht 11A $/ 11 \mathrm{D}=1 / 1$ ein, das man auch von 11D ausgehend erhält. Die Durchführung beider Isomerisierungen im präparativen Maßstab unter geringfügiger Modifikation $\left(45^{\circ} \mathrm{C}\right.$, Toluol) bestätigt das ${ }^{1} \mathrm{H}$-NMR-Experiment: Nach $3 \mathrm{~d}$ beträgt das Isomerenverhältnis 11A/11D annähernd $1 / 1$, die jeweiligen Startisomeren liegen nach dieser Zeit noch im leichten Überschuß vor. Das Isomer anti-11E konnte im ${ }^{1} \mathrm{H}$-NMR-Experiment nicht, bei der präparativen Durchführung nur in Spuren detektiert werden.

Aufgrund dieser Ergebnisse ist die Isomerisierung 11A $\rightarrow$ 11D als thermisch reversibel einzustufen (Gl. 1). Im Gegensatz hierzu liegt das Gleichgewicht für die pmcp-Komplexe nahezu quantitativ auf der Seite von Isomer D, wobei zusätzlich noch $\mathbf{E}$ entsteht [6]. Nicht entscheiden läßt sich, in welcher Reihenfolge die geschwindigkeitsbestimmenden Schritte, Öffnung der Sulfidbrücken (Übergangszustand B) bzw. S-S-Bindungsspaltung (Übergangszustand C) ablaufen [23]. Die Rückbildung terminaler zu Brücken-Schwefelliganden konnte bisher nur durch ihre Reaktion mit $\mathrm{H}_{2}, \mathrm{C}_{2} \mathrm{H}_{2}$ oder $\mathrm{C}_{2} \mathrm{H}_{4}$ [24,25] bzw. auf photochemischem Weg [26] erreicht werden. Auch die bei der Synthese von $\mathrm{L}_{2} \mathrm{Mo}_{2} \mathrm{~S}_{4}$ anfallenden blauen Lösungen von 16A und 17A isomerisieren, wenn auch unter vergleichweise drastischen Bedingungen. Nach $4 \mathrm{~d}$ bei $110^{\circ} \mathrm{C}$ haben sich im Fall von $\mathrm{L}=$ tbcp 16E (42\%), für $\mathrm{L}=$ ipcp syn-17D und anti-17E in $23 \mathrm{bzw} .17 \%$ Ausbeute gebildet. Eine exakte Bestimmung von etwaigen Gleichgewichten wird durch die Oligomerisierung der konzentrierten blauen Lösungen von 16A und 17A verhindert. Eindeutig ist dagegen die Umwandlung von syn-17D in anti-17E bei $110^{\circ} \mathrm{C}$. Anti-17E ist erwartungsgemäß [23] nicht isomerisierbar und wird unter diesen Bedingungen nahezu quantitativ zurückgewonnen. 


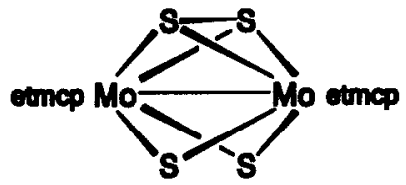

$11 \mathrm{~A}$

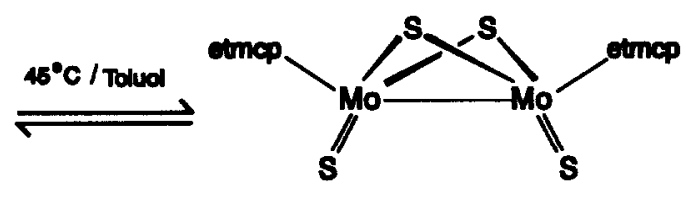

11D

\section{Experimenteller Teil}

Sämtliche Arbeiten wurden unter Schutzgas $\left(\mathrm{N}_{2}\right)$ und unter Verwendung von trockenen, $\mathrm{N}_{2}$-gesättigten Lösungsmitteln durchgeführt. Alle Molmassen wurden aus Toluollösung an einem Finnigan MAT95-Spektrometer bestimmt. Die ${ }^{1} \mathrm{H}$ - und ${ }^{95}$ Mo-NMR-Spektren wurden an einem Bruker-WM250-Spektrometer (externer Standard für ${ }^{95} \mathrm{Mo} \mathrm{Na}_{2} \mathrm{MoO}_{4}$, Meßtechnik nach [11]) aufgenommen. $\left(\mathrm{CH}_{3} \mathrm{CN}\right)_{3} \mathrm{Cr}$ $(\mathrm{CO})_{3}$ [27], t-Butyl- [28] und Ethyltetramethylcyclopentadien [29] wurden nach bereits beschriebenen Verfahren erhalten. Die Synthese von Isopropylcyclopentadien erfolgte analog zur Darstellung von t-Butylcyclopentadien aus $\mathrm{NaCp}$ und 2Brompropan.

\section{Allgemeine Vorschriften zur Darstellung der Komplexe 1-9}

Darstellung von $\mathrm{L}_{2} \mathrm{Cr}_{2}(\mathrm{CO})_{4}(\mathrm{~L}=t b c p$, ipcp) $(4,7)$

Eine Lösung von $\mathrm{ca} .13 \mathrm{mmol}\left(\mathrm{CH}_{3} \mathrm{CN}\right)_{3} \mathrm{Cr}(\mathrm{CO})_{3}$ und der äquimolaren Menge des jeweiligen Liganden in $c a .100 \mathrm{ml}$ Toluol wird $4 \mathrm{~h}$ unter Rückfluß erhitzt. Nach Einengen der grünen Reaktionslösung chromatographiert man an silanisiertem Kieselgel (Säule $22 \times 2 \mathrm{~cm}$ ). Mit Toluol eluiert man eine grüne Zone, die nach Abziehen des Lösungsmittels ein öliges Produkt liefert. Dieses gibt nach Überschichten mit etwa dem doppelten Volumen an Pentan bei $-18^{\circ} \mathrm{C}$ grüne, analysenreine Kristalle von 4 und 7 in 48 bzw. $42 \%$ Ausbeute.

Darstellung von etmcp ${ }_{2} \mathrm{Cr}_{2}(\mathrm{CO})_{4}$ (l) und $\mathrm{L}_{2} \mathrm{M}_{2}(\mathrm{CO})_{4}(\mathrm{~L}=\mathrm{etmcp}$, tbcp, ipcp; $M=M o, W)(2,3,5,6,8,9)$

$23 \mathrm{mmol}$ des entsprechenden Metallhexacarbonyls werden in ca. $60 \mathrm{ml}$-Decan suspendiert. Nach Zugabe einer äquimolaren Menge des jeweiligen Liganden erhitzt man $18 \mathrm{~h}$ unter Rückfluß. Nach dem destillativen Entfernen des Solvens löst man den Rückstand in möglichst wenig Toluol und chromatographiert an $\mathrm{SiO}_{2}$ (Säule $22 \times 3.5 \mathrm{~cm}$ ). Mit Toluol lassen sich für $\mathrm{M}=\mathrm{Cr}$ eine grüne, für $\mathbf{M}=\mathrm{Mo}$, W jeweils eine rote Zone eluieren. Die Komplexe 8 und 9 werden aus Pentan, 2, 3, 5 und 6 dagegen aus Toluol bei $-18^{\circ} \mathrm{C}$ umkristallisiert. Die Ausbeuten liegen zwischen 46 (9) und 65\% (2). C,H-Analysenwerte und FD-MS-Spektren entsprechen den berechneten Zusammensetzungen [14].

\section{Darstellung der Organometallsulfide 10-19}

Darstellung von etmcp ${ }_{2} \mathrm{Cr}_{2} \mathrm{~S}_{5}$ (10)

Dic grünc Lösung von $1.20 \mathrm{~g}(2.33 \mathrm{mmol})$ etmcp ${ }_{2} \mathrm{Cr}_{2}(\mathrm{CO})_{4}$ (1) und $374 \mathrm{mg}$ (11.66 mmol) Schwefel in $120 \mathrm{ml}$ Toluol wird $3 \mathrm{~d}$ bei $45^{\circ} \mathrm{C}$ gerührt. Die auf $c a .20$ 
ml konzentrierte Reaktionslösung chromatographiert man an $\mathrm{SiO}_{2}$. Zunächst eluiert man mit Toluol nicht umgesetztes Edukt 1 als grüne Zone (57\% Ausbeute) und dann mit Toluol/ Ether (5/1) eine weitere grüne Zone. Sie enthält 10 in $18 \%$ Ausbeute. Die Umkristallisation aus Toluol/Pentan (1/1) liefert dunkelgrüne Prismen. Elementaranalysen: 10: Gef.: C, 46.91; $\mathrm{H}, 5.90 . \mathrm{C}_{22} \mathrm{H}_{34} \mathrm{Cr}_{2} \mathrm{~S}_{5}(562.80)$ ber.: C, 46.95; H, 6.09\%. Molmasse 562.3 (Schwerpunkt).

Darstellung der Komplexe etmcp $\mathrm{Mo}_{2} \mathrm{~S}_{4}(\mathrm{HA}, \mathrm{D}, \mathrm{E})$

$1.81 \mathrm{~g}(3.00 \mathrm{mmol})$ etmcp $\mathrm{Mo}_{2}(\mathrm{CO})_{4}(2)$ und $250 \mathrm{mg}(7.80 \mathrm{mmol})$ Schwefel in $100 \mathrm{ml}$ Toluol läßt man $18 \mathrm{~h}$ bei $45^{\circ} \mathrm{C}$ reagieren. Die auf $c a .30 \mathrm{ml}$ eingeengte Reaktionslösung wird über Filterflocken filtriert und auf $\mathrm{SiO}_{2}$ aufgetragen (Säule $22 \times 3.5 \mathrm{~cm})$. Mit Toluol eluiert man als erste Zone wenig rotoranges etmcp ${ }_{2} \mathrm{Mo}_{2}(\mathrm{CO})_{6}$ und anschließend rotbraunes anti-etmcp ${ }_{2} \mathrm{Mo}_{2}(\mu-\mathrm{S})_{2} \mathrm{~S}_{2}(11 \mathrm{E})$ in $4 \%$ Ausbeute. Eine weitere braune Zone enthält $s y n$-etmcp ${ }_{2} \mathrm{Mo}_{2}(\mu-S)_{2} \mathrm{~S}_{2}(11 D)$ in 18\% Ausbeute. Mit Toluol/Ether (5/1) eluiert man schließlich blaues etmcp ${ }_{2} \mathrm{Mo}_{2}\left(\mu-\mathrm{S}_{2}\right)(\mu-\mathrm{S})_{2}(\mathbf{1 1 A})$ in einer Ausbeute von $21 \%$. Die Umkristallisation von 11A, D, E erfolgt aus Toluol. Elementaranalysen: 11A: Gef.: C, 42.82; H, 5.48. 11D: Gef.: C, 42.71; H, 5.42. 11E: Gef.: C, 42.89; H, 5.43. $\mathrm{C}_{22} \mathrm{H}_{34} \mathrm{Mo}_{2} \mathrm{~S}_{4}(618.62)$ ber.: C, 42.71 ; H, 5.54\%. Molmassen: 11A: 610.5 (bezogen auf ${ }^{92} \mathrm{Mo}$ ). 11D: 610.3 (bezogen auf ${ }^{92} \mathrm{Mo}$ ). 11E: 610.4 (bezogen auf ${ }^{92} \mathrm{Mo}$ ).

\section{Umsetzung von etmcp ${ }_{2} \mathrm{~W}_{2}(\mathrm{CO})_{4}(3)$ mit Schwefel}

Eine Mischung aus $1.56 \mathrm{~g}(2.00 \mathrm{mmol}) 3,256 \mathrm{mg}(8.00 \mathrm{mmol})$ Schwefel und 120 $\mathrm{ml}$ Toluol wird $16 \mathrm{~h}$ bei $45^{\circ} \mathrm{C}$ gerührt. Die nunmehr grüne Reaktionslösung wird nach Einengen auf $c a .20 \mathrm{ml}$ an $\mathrm{SiO}_{2}$ chromatographiert. Mit Toluol eluiert man zunächst eine grüne $\left(\operatorname{etmcp}_{2} \mathrm{~W}_{2}(\mathrm{CO})_{2} \mathrm{~S}_{3}(12), 56 \%\right.$ Ausbeute) und dann eine dunkelgrüne Zone (syn-etmcp, $\mathrm{W}_{2}(\mu-\mathrm{S})_{2} \mathrm{~S}_{2}$ (13D), 12\% Ausbeute, analytische Daten s.u.). Elementaranalysen: 12: Gef.: C, 35.67; H, 4.02. $\mathrm{C}_{24} \mathrm{H}_{34} \mathrm{~W}_{2} \mathrm{O}_{2} \mathrm{~S}_{3}(818.41)$ ber.: C, 35.22; H, 4.19\%. Molmasse 818.7 (Schwerpunkt).

\section{Darstellung von etmcp $\mathrm{W}_{2} S_{4}(13 D, E)$ aus 12}

Die grüne Lösung von $800 \mathrm{mg}(0.98 \mathrm{mmol})$ etmcp $\mathrm{W}_{2}(\mathrm{CO})_{2} \mathrm{~S}_{3}(12)$ in $80 \mathrm{ml}$ Toluol wird mit $35 \mathrm{mg}(1.10 \mathrm{mmol})$ Schwefel versetzt. Nach $15 \mathrm{~h}$ Rühren bei $110^{\circ} \mathrm{C}$ engt man die jetzt braune Reaktionslösung auf $c a .20 \mathrm{ml}$ ein und chromatographiert an $\mathrm{SiO}_{2}$. Mit Toluol läßt sich zuerst braunes anti-etmcp ${ }_{2} \mathrm{~W}_{2}(\mu-\mathrm{S})_{2} \mathrm{~S}_{2}(13 E)$ in $58 \%$ und dann grünes syn-etmcp ${ }_{2} \mathrm{~W}_{2}(\mu-\mathrm{S})_{2} \mathrm{~S}_{2}(13 \mathrm{D})$ in $21 \%$ Ausbeute eluieren. Beide Isomere werden aus Toluol umkristallisiert. Elementaranalysen: 13D: Gef.: C, 33.37; H, 4.30. 13E: Gef.: C, 33.97; $\mathrm{H}, 4.33 . \mathrm{C}_{22} \mathrm{H}_{34} \mathrm{~W}_{2} \mathrm{~S}_{4}$ (794.45) ber.: C, 33.26; H, 4.31\%. Molmassen: 13D: 794.0 (Schwerpunkt). 13E: 794.0 (Schwerpunkt).

Umsetzungen von $L_{2} \mathrm{Mo}_{2}(C O)_{4}(L=t b c p 5$, ipcp 8) mit Schwefel

(a) Stöchiometrie Mo $/ S=1 / 1$. $1.0 \mathrm{mmol} 5 \mathrm{bzw} .8$ werden mit $64 \mathrm{mg}(2.0$ mmol) Schwefel in $80 \mathrm{ml}$ Toluol gelöst und $4 \mathrm{~h}$ bei $45^{\circ} \mathrm{C}$ gerührt. Die konzentrierte Reaktionslösung wird an $\mathrm{SiO}_{2}$ chromatographiert, wobei mit Toluol die violetten Komplexe $\mathrm{L}_{4} \mathrm{Mo}_{4} \mathrm{~S}_{4}$ in $62 \%$ (14) bzw. $56 \%$ Ausbeute (15) eluiert werden. Die Umkristallisation von 14 und 15 erfolgt aus Toluol. Elementaranalysen: 14: Gef.: C, 43.23; H, 5.21. $\mathrm{C}_{36} \mathrm{H}_{52} \mathrm{Mo}_{4} \mathrm{~S}_{4}(996.81)$ ber.: C, 43.38; $\mathrm{H}, 5.26 \%$. Molmasse 996.9 
(Schwerpunkt). 15: Gef.: C, 41.13; H, 4.63. $\mathrm{C}_{32} \mathrm{H}_{44} \mathrm{Mo}_{4} \mathrm{~S}_{4}$ (940.70) ber.: C, 40.86; H, 4.71\%. Molmasse 941.0 (Schwerpunkt).

(b) Stöchiometrie $M o / S=1 / 2$. Die rote Lösung von $1.0 \mathrm{mmol} 5$ bzw. 8 und $128 \mathrm{mg}(4.0 \mathrm{mmol})$ Schwefel in $80 \mathrm{ml}$ Toluol wird $7 \mathrm{~h}$ bei $45^{\circ} \mathrm{C}$ gerührt. Die nunmehr blaue Lösung wird auf $c a$. die Hälfte eingeengt und an $\mathrm{SiO}_{2}$ (Säule $25 \times 3 \mathrm{~cm})$ chromatographiert. Zunächst eluiert man mit Toluol rotbraunes anti16E (6\% Ausbeute) bzw. anti-17E (14\%) und dann braunes 17D (9\%). Mit Toluol/Ether (10/1) folgt in beiden Fällen eine blaue Zone, die nach dem Entfernen des Lösungsmittels cinen braunen Feststoff gibt, dem versuchsweise die Zusammensetzung $\left[\mathrm{L}_{2} \mathrm{Mo}_{2} \mathrm{~S}_{4}\right]_{n}$ zugeschrieben wird. Bezogen auf "tbcp $\mathrm{Mo}_{2} \mathrm{~S}_{4}$ " bzw. " $\mathrm{ipcp}_{2} \mathrm{Mo}_{2} \mathrm{~S}_{4}$ " würden die Ausbeuten 68 bzw. $57 \%$ betragen. Elementaranalysen: 16E: Gef.: C, 38.71; $\mathrm{H}, 4.08 . \mathrm{C}_{18} \mathrm{H}_{26} \mathrm{Mo}_{2} \mathrm{~S}_{4}(562.52)$ ber.: $\mathrm{C}, 38.43 ; \mathrm{H}, 4.66 \%$. Molmasse 554.0 (bezogen auf ${ }^{92} \mathrm{Mo}$ ). 17D: Gef.: $\mathrm{C}, 35.85$; H, 4.04. 17E: Gef.: C, 35.83; H, 4.00. $\mathrm{C}_{16} \mathrm{H}_{22} \mathrm{Mo}_{2} \mathrm{~S}_{4}$ (534.47) ber.: C, 35.96; H, 4.15\%. Molmassen: 17D: 526.0 (bezogen auf ${ }^{92} \mathrm{Mo}$ ). 17E: 526.0 (bezogen auf ${ }^{92} \mathrm{Mo}$ ).

\section{Darstellung von $L_{2} W_{2} S_{4}(L=t b c p 18 D, E ;$ ipcp 19D, E)}

Das Gemisch aus $1.0 \mathrm{mmol} 6(9), 128 \mathrm{mmol}(4.0 \mathrm{mmol})$ Schwefel und $100 \mathrm{ml}$ Toluol wird $18 \mathrm{~h}$ bei $50^{\circ} \mathrm{C}$ gerührt. Nach dem Einengen auf ca. $30 \mathrm{ml}$ wird von ausgefallenem $\mathrm{L}_{2} \mathrm{~W}_{2}(\mathrm{CO})_{6}$ abfiltriert und dann an $\mathrm{SiO}_{2}$ mit Toluol als Laufmittel chromatographiert (Säule $3.5 \times 20 \mathrm{~cm}$ ). Zunächst eluiert man Reste von organgerotem $\mathrm{L}_{2} \mathrm{~W}_{2}(\mathrm{CO})_{6}$, vor einer rotbraunen und einer grünbraunen Zone. Erstere enthält anti- $\mathrm{L}_{2} \mathrm{~W}_{2}(\mu-\mathrm{S})_{2} \mathrm{~S}_{2}(18 \mathrm{E})$ in $22 \%(19 \mathrm{E}: 18 \%)$, letztere $s y n-\mathrm{L}_{2} \mathrm{~W}_{2}(\mu$ S) $\mathrm{S}_{2}$ (18D) in 28\% Ausbeute (19D: 24\%). Die Umkristallisation von 18D, $\mathrm{E}$ und 19D, E erfolgt aus Toluol. Elementaranalysen: 18D: Gef.: C, 29.41; H, 3.51. 18E: Gef.: C, 29.49; H, 3.48. $\mathrm{C}_{18} \mathrm{H}_{26} \mathrm{~W}_{2} \mathrm{~S}_{4}$ (738.34) ber.: C, 29.28; H, 3.55\%. Molmassen: 18D: 738.5 (Schwerpunkt). 18E: 738.5 (Schwerpunkt). 19D: Gef.: C, 27.09; H, 3.03. 19E: Gef.: C, 27.28; H, 3.05. $\mathrm{C}_{16} \mathrm{H}_{22} \mathrm{~W}_{2} \mathrm{~S}_{4}$ (710.29) ber.: C, $27.06 ; \mathrm{H}, 3.12 \%$. Molmassen: 19D: 710.0 (Schwerpunkt). 19E: 710.0 (Schwerpunkt).

Untersuchungen zur thermischen Stabilität von $L_{2} \mathrm{Mo}_{2}\left(\mu, \eta^{2}-S_{2}\right)(\mu-S)_{2}(L=e t m c p$, ipcp)

Isomerisierung von etmcp $\mathrm{Mo}_{2} \mathrm{~S}_{4}(11 \mathrm{~A}, \mathrm{D}, \mathrm{E})$. Die blaue Lösung von $760 \mathrm{mg}$ $(1.23 \mathrm{mmol}) 11 \mathrm{~A}$ in $80 \mathrm{ml}$ Toluol wird $3 \mathrm{~d}$ bei $45^{\circ} \mathrm{C}$ gerührt. Nach Einengen auf $c a$. $20 \mathrm{ml}$ chromatographiert man an $\mathrm{SiO}_{2}$. Mit Toluol wird braunes $11 \mathrm{D}$ in $43 \%$, mit Toluol/Ether (5/1) blaues Edukt in 51\% Ausbeute cluicrt. Isomerisiert man unter den gleichen Bedingungen braunes 11D, so erhält man blaues 11A in $40 \%$ Ausbeute, während $46 \%$ an 11D zurückgewonnen werden. In beiden Fällen lassen sich nur Spuren von $11 E$ beobachten.

Isomerisierung von ipcp ${ }_{2} \mathrm{Mo}_{2}\left(\mu, \eta^{2}-S_{2}\right)(\mu-S)_{2} \quad(17 \mathrm{~A})$. Das blaue, 17A enthaltende Toluoleluat aus der Darstellung von ipcp ${ }_{2} \mathrm{Mo}_{2} \mathrm{~S}_{4}$ wird $4 \mathrm{~d}$ bei $110^{\circ} \mathrm{C}$ gerührt. Anschließend chromatographiert man die auf die Hälfte konzentrierte Lösung an $\mathrm{SiO}_{2}$, wobei die löslichen Komplexe 17D, E mit Toluol eluiert werden. Die mit Toluol/Ether (10/1) eluierte blaue Zone wandelt sich beim Konzentrieren in braunes, schwerlösliches [ipcp $\left.{ }_{2} \mathrm{Mo}_{2} \mathrm{~S}_{4}\right]_{\mathrm{n}}$ um. Die relative Ausbeute an letzterem beträgt $60 \%$, während 17D, E in 17 bzw. 23\% Ausbeute entstehen. Ein mit 17D durchgeführtes Kontrollexperiment unter analogen Bedingungen liefert nur Hinweise auf die Bildung von 17E (34\% Ausbeute). 
Röntgenographische Daten von syn-etmcp ${ }_{2} \mathrm{Mo}_{2}(\mu-S)_{2} \mathrm{~S}_{2}$ (IID)

Schwarzer Kristall $\left(0.20 \times 0.20 \times 0.80 \mathrm{~mm}^{3}\right)$, rhombisch $D_{2 \mathrm{~h} / 15}, P b c a(61)$; Zellkonstanten: $a$ 10.918(3), $b$ 15.458(4), $c$ 30.19(1) $\AA ; V$ 5095.20 $\AA^{3}, Z=8$; empirische Absorptionskorrektur (psi-scan Messung: 7 Reflexe $5.3<2 \theta<46.1^{\circ}$. Transmiss. Faktor (min./max.) 0.775/0.953), $\mu 1.28 \mathrm{~mm}^{-1}$. $F(000) 2512, d$ (röntg) $=1.61$ $\mathrm{g} / \mathrm{cm}^{3}$; Syntex R3. Mo- $K_{\alpha}-$ Strahlung, Graphit-Monochromator, im vermessenen Bereich 8195 mögliche Reflexe; unabhängige Reflexe $4792(I>2.5 \sigma(I))$. Die Struktur wurde gelöst mittels Patterson-, Fourier- und Differenzfouriersynthesen; die H-Atome wurden mit Hilfe des SHELXTL-Unterprogramms HFIX [30] fixiert; $R=0.048, R_{\mathrm{w}}=0.040 ;$ Restelektronendichte $(\max . / \min ) 0.63 /.-0.55 \mathrm{e} / \AA^{3}$, shift /esd $(\operatorname{mean} / \max )-./ 0.013, G O O F=2.16$.

Röntgenographische Daten von syn-etmcp ${ }_{2} W_{2}(\mu-S)_{2} S_{2}(13 D)$

Grünbrauner Kristall $\left(0.20 \times 0.22 \times 0.80 \mathrm{~mm}^{3}\right)$, rhombisch $D_{2 \mathrm{~h} / 15}, P b c a$ (61); Zellkonstanten: $a$ 10.905(2), $b$ 15.485(3), c 30.091(6) $\AA$; $V$ 5081.3 $\AA^{3}, Z=8$; empirische Absorptionskorrektur (psi-scan Messung: 8 Reflexe $5.4<2 \theta<51.0^{\circ}$. Transmiss. Faktor (min./max.) 0.785/1.000), $\mu 9.57 \mathrm{~mm}^{-1} . F(000) 3024, d$ (röntg) $=2.08 \mathrm{~g} / \mathrm{cm}^{3}$; Syntex R3. Mo- $K_{\alpha}$-Strahlung, Graphit-Monochromator, im vermessenen Bereich 8180 mögliche Reflexe; unabhängige Reflexe $4727(I>2.5 \sigma(I))$. Die Struktur wurde gelöst mittels Patterson-, Fourier- und Differenzfouriersynthesen; die H-Atome wurden mit Hilfe des SHELXTL-Unterprogramms HFIX [30] fixiert; $R=0.046, R_{\mathrm{w}}=0.039 ;$ Restelektronendichte (max. $/ \mathrm{min}$.) $1.38 /-1.38$ $\mathrm{e} / \AA^{3}$, shift $/$ esd (mean $/ \max$.) $-/ 0.09$, GOOF $=1.78$

\section{Dank}

Wir danken Herrn Dr. T. Burgemeister für die Aufnahme der ${ }^{95}$ Mo-Spektren und dem massenspektroskopischen Labor der Universität Regensburg für die Aufnahme der Massenspektren.

\section{Literatur}

1 E.I. Stiefel, T.R. Halbert, C.L. Coyle, L. Wei, W.-H. Pan, T.C. Ho, R.R. Chianelli und M. Daage, Polyhedron, 8 (1989) 1625.

2 M. Rakowski DuBois, Chem. Rev., 89 (1989) 1.

3 G.J. Kubas, R.R. Ryan, K.A. Kubat-Martin und E. Larson, J. Am. Chem. Soc., 111 (1989) 7823.

4 J. Wachter, Angew. Chem., 101 (1989) 1645; Angew. Chem., Int. Ed. Engl., 28 (1989) 1613.

5 H. Brunner, J. Wachter, F. Guggolz, M.L. Ziegler und B. Balbach, J. Am. Chem. Soc., 104 (1982) 1765 .

6 H. Brunner, W. Meier, J. Wachter, E. Guggolz, T. Zahn und M.L. Ziegler, Organometallics, 1 (1982) 1107.

7 M. Rakowski DuBois, M.C. Van Derveer, D.L. DuBois und R.C. Haltiwanger, Inorg. Chem., 20 (1981) 3064.

8 M.D. Curtis und W.M. Butler, J. Chem. Soc., Chem. Commun., (1980) 998.

9 R.B. King, M.Z. Iqbal und A.D. King Jr., J. Organomet. Chem., 6 (1987) 1051.

10 R.J. Klingler, W.M. Butler und M.D. Curtis, J. Am. Chem. Soc., 100 (1978) 5034; M.D. Curtis, L. Messerle, N.A. Fotinos und R.F. Gerlach, ACS Symp. Ser., 155 (1981) 221.

11 C.G. Young, M. Minelli, J.H. Enemark, G. Miessler, N. Janietz, H. Kauermann und J. Wachter, Polyhedron, 5 (1986) 407.

12 C.J. Casewitt, D.E. Coons, L.L. Wright, W.K. Miller und M. Rakowski DuBois, Organometallics, 5 (1986) 951. 
13 Tatsächlich lassen sich die braunen Feststoffe mit $\mathrm{LiEt}_{3} \mathrm{BH}$ in die löslichen Komplexe $\mathrm{L}_{2} \mathrm{Mo}_{2}(\mu$ $\mathrm{SH})_{2}(\mu-\mathrm{S})_{2}$ überführen [14].

14 R. Graß1, Dissertation Universität Regensburg, 1991.

15 L.D. Tanner, R.C. Haltiwanger und M. Rakowski DuBois, Inorg. Chem., 27 (1988) 1741.

16 J.A. Bandy, C.E. Davies, J.C. Green, M.L.H. Green, K. Prout und D.P.S. Rodgers, J. Chem. Soc., Chem. Commun., (1983) 1395.

17 C.J. Casewitt, M. Rakowski DuBois, R.A. Grieves und J. Mason, Inorg. Chem., 26 (1987) 1889.

18 J.C. Green, R.A. Grieves und J. Mason, J. Chem. Soc., Dalton Trans., (1986) 1313.

19 O.A. Rajan, M. McKenna, J. Noordik, R.C. Haltiwanger und M. Rakowski DuBois, Organometallics, 3 (1984) 831.

20 F. Secheresse, J. Lifebore, J.C. Daran und Y. Jeannin, Inorg. Chim. Acta, 45 (1980) L 45.

21 M.G.B. Drew, E.M. Page und D.A. Rice, Inorg. Chim. Acta, 76 (1983) L 33.

22 A. Bino, F.A. Cotton, Z. Dori und J.C. Sekutowski, Inorg. Chem., 17 (1978) 2946.

23 W. Tremel, R. Hoffmann und E.D. Jemmis, Inorg. Chem., 28 (1989) 1213; B.E. Bursten und R.H. Cayton, ibid., 28 (1989) 2846.

24 M. Rakowski DuBois, M.C. Van Derveer, D.L. DuBois, R.C. Haltiwanger und W.K. Miller, J. Am. Chem. Soc., 102 (1980) 7456.

25 D.L. DuBois, W.K. Miller und M. Rakowski DuBois, J. Am. Chem. Soc., 103 (1981) 3429.

26 A.E. Bruce und D.R. Tyler, Inorg. Chem., 23 (1984) 3433.

27 G.J. Kubas und L.S. van der Sluys, Inorg. Synth., 28 (1991) 29.

28 R. Riemschneider, A. Reisch und H. Horak, Monatsh. Chem., 91 (1960) 52.

29 R.S. Threlkel und J.E. Bercaw, J. Organomet. Chem., 136 (1977) 1.

30 G.M. Sheldrick, SHELXTL-Program (Unterprogramm HFIX), Universität Göttingen, Deutschland, 1983. 